\title{
Efficacy of alendronate for the treatment of ankylosing spondylitis: a protocol for systematic review and meta-analysis
}

\section{Yu-hua Tang}

First Affiliated Hospital of Jiamusi University

Yu-zhi Li

First Affiliated Hospital of Jiamusi University

Zhao-chen Tang

Jiamusi University

Quan-wei Jiang

Benxi Central Hospital of China Medical University

Yu Zhao ( $\nabla$ taoyu200101@outlook.com )

Huludao Central Hospital

\section{Protocol}

Keywords: Ankylosing spondylitis, alendronate, efficacy, safety

Posted Date: May 14th, 2020

DOl: https://doi.org/10.21203/rs.3.rs-27663/v1

License: (c) (i) This work is licensed under a Creative Commons Attribution 4.0 International License. Read Full License

Version of Record: A version of this preprint was published at Medicine on July 24th, 2020. See the published version at https://doi.org/10.1097/MD.0000000000021089. 


\section{Abstract}

Background Ankylosing spondylitis (AS) is a very tricky orthopedic disorder. If such condition can not be managed fairly well, it may significantly affect quality of life and even leads to disability among such population. A variety of studies have reported that alendronate is utilized for the treatment of AS. However, their results are still contrary, and no systematic review has addressed on this topic. Thus, this study will systematically assess the efficacy and safety of alendronate for the treatment of patients with AS.

Methods A comprehensive literature search will be performed from the below electronic databases from their commencement to the January 31, 2020 without language and publication status limitations: PUBMED, EMBASE, Cochrane Library, Web of Science, Allied and Complementary Medicine Database, WANGFANG, and China National Knowledge Infrastructure. Only randomized controlled trials (RCTs) focusing on the alendronate for the treatment of patients with AS will be considered for inclusion in this study. Two authors will independently select all identified records, extract essential data from all included studies, and appraise study quality for each eligible trial using Cochrane risk of bias. If any differences occur, another experienced author will be invited to solve them by discussion and a consensus decision will be made. We will implement RevMan 5.3 software to analyze the extracted data.

Results This study will summarize high quality RCTs to assess the efficacy and safety of alendronate for the treatment of patients with AS through primary outcome of bone densitometry; and secondary outcomes of pain intensity, quality of life, disease activity, functional status, and adverse events.

Conclusions This study will provide evidence to help determine whether alendronate is an effective and safe management for patient with AS or not.

\section{Background}

Ankylosing spondylitis (AS) is a chronic inflammatory rheumatic disease [1-3]. It is characterized by inflammation of spine and the sacroiliac joints [4-6], which causes structural and functional impairments of those joints and results in poor quality of life [7-9]. It is estimated that about $0.1 \%$ to $1.8 \%$ people affect this disorder and onset of symptoms commonly manifest in males between 15 and 25 years old [10-11]. To date, its etiology remains not fully elucidated [12-13].

Currently, very few medications can benefit patients with AS [14-16]. Recent studies reported to use alendronate for the treatment of patients with AS [17-23]. As far as we know, no previous systematic review has focused on the efficacy and safety of alendronate for treating AS. With the recent publication 
of high quality studies on this subject [17-23], this study aims to systematically explore the efficacy and safety of alendronate for the treatment of patients with AS.

\section{Methods And Analysis}

\section{Study registration}

We have registered this study on INPLASY202040153. It is developed according to the Preferred Reporting Items for Systematic Reviews and Meta-Analysis Protocol statement guidelines [24].

\section{Eligibility criteria for study selection}

\section{Study types}

This study will only include randomized controlled trials (RCTs) of alendronate for the treatment of patients with AS. Besides RCTs, we will exclude any other studies, such as non-clinical trials, non-RCTs, and quasi-RCTs.

\section{Intervention types}

The experimental intervention are any forms of alendronate.

The control intervention could be any treatments, such as placebo, other medications, and alternative therapies. However, we will exclude studies using any types of alendronate, including its combination with other managements as their comparators.

\section{Patient types}

Any adult participants (more than 18 years old) with a definite diagnosis of AS will be included in spite of their race, gender, educational background, and duration of AS.

\section{Outcome measurements}

\section{Primary outcome}

Bone densitometry (as reported in the trials by any instruments or tools). 


\section{Secondary outcomes}

Pain intensity (as reported in the trial by any pain scales);

Quality of life (as reported in the trial by any tools, such as Ankylosing Spondylitis Quality of Life questionnaire);

Disease activity (as reported in the trial by any indexes, such as Bath Ankylosing Spondylitis Disease Activity Index);

Functional status (as reported in the trial by any scores, such as Bath Ankylosing Spondylitis Functional Index); and

Adverse events.

\section{Search strategy}

\section{Electronic databases search}

Two authors will carry out a comprehensive literature search from the below electronic databases: PUBMED, EMBASE, Cochrane Library, Web of Science, Allied and Complementary Medicine Database, WANGFANG, and China National Knowledge Infrastructure. All these electronic databases will be searched from their commencement to the January 31, 2020 without language and publication status limitations. Any potential randomized controlled trials (RCTs) that explored the efficacy and safety of alendronate for the treatment of patients with AS will be included. An example of detailed search strategy for PUBMED is presented (table 1). We will modify similar search strategies for other electronic databases.

\section{Other literature sources search}

We will search other literature resources, such as Google Scholar, websites of clinical trial registry, dissertations, and reference lists of included trials.

\section{Literature selection}

Records from all literature source searches will be imported into EndNote X9 to remove all duplicated studies. Two authors will firstly identify records by scanning titles and abstracts, and unrelated studies 
will be eliminated. Then, full-text of remaining studies will be read cautiously based on the predefined eligibility criteria. Any divergences will be figured out with the help of a third author. The process of study selection will be exerted in a flow diagram (Figure 1).

\section{Data collection and management}

Two authors will independently extract data from each included trial in accordance with the predefined data collection form. Any uncertainties will be resolved by another author and a consensus will be reached finally. The collected information is as follows:

Study details: title, first author, publication date, location, et al.

Study methods: randomization specifics, blinding, allocation, follow-up information, et al.

Patient characteristics: age, sex, sample size, duration and severity of AS, et al.

Intervention and control details: treatment duration, dosage, frequency, et al.

Outcomes: outcome measurements reported in the trial, adverse events, et al.

Others: funding information, conflict of interest, et al.

\section{Dealing with unclear or missing data}

Any unclear or insufficient data will be obtained from original authors with email or phone. If it is not achievable, we will perform data analysis based on the available data, and will discuss its impacts on study findings.

\section{Risk of bias assessment}

Study quality of each included trial will be evaluated by two independent authors using Cochrane risk of bias tool, which examines 7 domains. We will rate each source of bias as low, unclear or high. Any different views will be worked out with the help of another author through discussion.

\section{Treatment effect measurement}

In this study, we will use mean difference or standardized mean difference and $95 \%$ confidence intervals (Cls) to evaluate the treatment effect for continuous data, and risk ratio and $95 \% \mathrm{Cls}$ to appraise the 
treatment effect for dichotomous data.

\section{Data analysis}

RevMan 5.3 software will be employed for all data analysis and meta-analysis if it is possible. $I^{2}$ test will be used for heterogeneity identification. $I^{2} \leq 50 \%$ suggests reasonable heterogeneity, and a fixed-effect model will be exerted. Otherwise, $I^{2}>50 \%$ means substantial heterogeneity, and a random-effect model will be employed. If ample data is extracted from sufficient trials with low heterogeneity, we will carry out meta-analysis to explain the results. On the other hand, we will perform subgroup analysis and sensitivity analysis to test possible sources of significant heterogeneity. If meta-analysis is deemed not to be conducted, we will elaborate study results using a narrative description.

\section{Subgroup analysis}

Subgroup analysis will be developed to investigate the possible sources of significant heterogeneity according to the characteristics of study, types of intervention and controls, and different outcome measurements.

\section{Sensitivity analysis}

A sensitivity analysis will be carried out to test the robustness and stability of pooled results by taking away low quality trials or trials reporting data missing.

\section{Reporting bias}

If it is possible to include at least 10 trials on one outcome, we will examine the possible reporting bias using funnel plot and Egger's regression test [25-26].

\section{Dissemination}

This study will be disseminated at a peer-reviewed journal or will be presented a scientific conference.

\section{Discussion}


Alendronate is used to treat several osteoporosis diseases, including AS. For patients with AS, alendronate has been investigated in several clinical trials. However, its efficacy and safety on AS is still inconsistent. Hence, this study will assess the efficacy and safety of alendronate in patients with AS. This study may provide helpful evidence to judge whether alendronate is effective and safety in patients with AS.

This study still has several limitations. First, significant heterogeneity may exist, which may affect the accuracy of the findings. Second, AS severity and baseline characteristics of the patients may be vary among trials, this may impede its generation to all AS patients. Third, the number of eligible trials and patient samples may be relatively small, which may weaken the power of the present study.

\section{Abbreviations}

AS, ankylosing spondylitis; RCTs, randomized controlled trials; Cls, confidence intervals.

\section{Declarations}

Ethics approval and consent to participate: Not applicable

Consent for publication: Not applicable

Availability of data and material: Data sharing is not applicable to this article as no datasets were generated or analyzed during the current protocol

Competing interests: None.

Funding: This study was supported by Scientific Research Project of Heilongjiang Health and Family Planning Commission (2017-387). The supporters had no role in this study.

Authors' contributions: YHT and YZ conceived the study. YZL and ZCT contributed with the clinical background and expertise. YZL, ZCT and QWJ contributed with the analytical plan and the bias 
assessment approach. YHT and YZL performed the literature search plan. ZCT, QWJ and YZ drafted the protocol. All authors revised the protocol and approved the final version. YZ supervised the study

Acknowledgements: Not applicable.

Authors' information: Yu-hua Tang, huayut@outlook.com

Yu-zhi Li, xuancang8173@126.com

Zhao-chen Tang, baoliaoyue794393@126.com

Quan-wei Jiang, neanenmjuqqg@outlook.com

Yu Zhao, Yuzhao2001@outlook.com

\section{References}

1. Li M, Zhou X, Zhou L, Yu Z, Fu L, Yang P. Meta-Analysis of Changes in the Number and Proportion of Regulatory T Cells in Patients with Ankylosing Spondylitis. Biomed Res Int 2020; 2020:8709804.

2. Xi Y, Jiang T, Chaurasiya $B$, et al. Advances in nanomedicine for the treatment of ankylosing spondylitis. Int J Nanomedicine 2019; 14:8521-42.

3. Lai NL, Zhang SX, Wang J, et al. The Proportion of Regulatory T Cells in Patients with Ankylosing Spondylitis: A Meta-Analysis. J Immunol Res 2019; 2019:1058738.

4. Zhao J, Huang $\mathrm{C}$, Huang $\mathrm{H}$, et al. Prevalence of ankylosing spondylitis in a Chinese population: a systematic review and meta-analysis. Rheumatol Int 2020; doi: 10.1007/s00296-020-04537-0. (Epub ahead of print)

5. Mitsui H. Ankylosing Spondylitis. Clin Calcium 2008; 18(12):1781-5.

6. Dean LE, Jones GT, MacDonald AG, Downham C, Sturrock RD, Macfarlane GJ. Global prevalence of ankylosing spondylitis. Rheumatology (Oxford) 2014; 53(4):650-7.

7. Raychaudhuri SP, Deodhar A. The classification and diagnostic criteria of ankylosing spondylitis. J Autoimmun 2014; 48-49:128-33.

8. Zochling J, Braun J. Mortality in rheumatoid arthritis and ankylosing spondylitis. Clin Exp Rheumatol 2009; 27(4 Suppl 55):S127-30.

9. Chee MM, Sturrock RD. Ankylosing spondylitis. Scott Med J 2007; 52(4):32-5.

10. He C, He X, Tong W, et al. The effect of total hip replacement on employment in patients with ankylosing spondylitis. Clin Rheumatol 2016; 35(12):2975-81. 
11. Feldtkeller E, Khan MA, van der Heijde D, van der Linden S, Braun J. Age at disease onset and diagnosis delay in HLA-B27 negative vs. positive patients with ankylosing spondylitis. Rheumatol Int 2003; 23(2):61-6.

12. Braun J, Sieper J. Ankylosing spondylitis. Lancet 2007; 369(9570):1379-90.

13. Laitinen M, Hakala M. Ankylosing spondylitis. Duodecim 2005; 121(15):1635-42.

14. Murata H. Ankylosing spondylitis. Nihon Rinsho 2002; 60 (Suppl 1):411-6.

15. van der Horst-Bruinsma IE, Clegg DO, Dijkmans BA. Treatment of ankylosing spondylitis with disease modifying antirheumatic drugs. Clin Exp Rheumatol 2002; 20(6 Suppl 28):S67-70.

16. Cai G, Wang L, Fan D, et al. Vitamin D in ankylosing spondylitis: review and meta-analysis. Clin Chim Acta $2015 ;$ 438:316-22.

17. Lewis $G$, Janna S. Alendronate in bone cement: fatigue life degraded by liquid, not by powder. Clin Orthop Relat Res 2006; 445:233-8.

18. Liang SF. The therapeutic effect of alendronate combined with calcium Erqi D on elderly female patients with type 2 diabetic osteoporosis. Strait Pharmacy 2019; 31 (5): 129-31.

19. Dong W, Qi MC, Liu Q, Yu J, Deng JP. The effect of alendronate on osteoclastogenesis and bone resorption function at different stages of differentiation. Journal of Stomatology 2011; 27 (3): 195-8.

20. Jia $\mathrm{P}, \mathrm{Tang} \mathrm{H}$. The role of alendronate in reducing the risk of osteoporotic vertebral fracture. Chinese Journal of Osteoporosis 2008; (8): 591-5.

21. Li G, Lv CA, Tian L, Jin LJ, Zhao W. A retrospective study of alendronate for the treatment of ankylosing spondylitis. Medicine (Baltimore) 2018; 97(20):e10738.

22. Coates L, Packham JC, Creamer P, et al. Clinical efficacy of oral alendronate in ankylosing spondylitis: a randomised placebo-controlled trial. Clin Exp Rheumatol 2017; 35(3):445-51.

23. Khabbazi A, Noshad H, Gafarzadeh S, Hajialiloo M, Kolahi S. Alendronate Effect on the Prevention of Bone loss in Early Stages of Ankylosing Spondylitis: A Randomized, Double-Blind, Placebo-Controlled Pilot Study. Iran Red Crescent Med J 2014; 16(6):e18022.

24. Moher $D$, Shamseer $L$, Clarke $M$, et al. Preferred reporting items for systematic review and metaanalysis protocols (PRISMA-P) 2015 statement. Syst Rev 2015; 4:1.

25. Sutton AJ, Duval SJ, Tweedie RL, et al. Empirical assessment of effect of publication bias on metaanalyses. BMJ 2000; 320:1574-7.

26. Egger M, Davey Smith G, Schneider M, et al. Bias in meta-analysis detected by a simple, graphical test. BMJ 1997; 315:629-34.

\section{Tables}

Table 1 Search strategy of PUBMED 


\begin{tabular}{ll}
\hline Number & Search terms \\
\hline 1 & ankylosing spondylitis \\
2 & Bechterew's disease \\
3 & uveitis \\
4 & joint stiffness \\
5 & inflammation \\
6 & spine \\
7 & arthritis \\
8 & Or 1-7 \\
9 & alendronate \\
10 & Binosto \\
11 & Fosamax Plus D \\
12 & Fosamax \\
13 & Or 9-12 \\
14 & randomized controlled trials \\
15 & random \\
16 & control \\
17 & allocation \\
18 & placebo \\
19 & clinical trial \\
20 & controlled study \\
21 & Or 14-20 \\
22 & 8 and 13 and 21 \\
\hline
\end{tabular}

\section{Supplementary Files}

This is a list of supplementary files associated with this preprint. Click to download.

- PRISMAPchecklist.doc 